\title{
Wogonin induces apoptosis and endoplasmic reticulum stress in HL-60 leukemia cells through inhibition of the PI3K-AKT signaling pathway
}

\author{
CHENGJUN HU*, MAOZHONG XU*, RUJUAN QIN, WEIFENG CHEN and XIN XU \\ Department of Hematology, The Affiliated Jiangyin Hospital \\ of Southeast University Medical College, Jiangyin, Jiangsu 214400, P.R. China
}

Received December 19, 2014; Accepted March 17, 2015

DOI: $10.3892 /$ or.2015.3896

\begin{abstract}
Wogonin is a flavonoid isolated from Scutellaria baicalensis root and has multiple pharmacological effects, including anticancer effects. Recent studies have shown that wogonin induces cell cycle arrest and reverses multi-drug resistance in the human K562 leukemia cell line. However, its pharmacological function in the apoptosis of leukemia cells remains unknown. Therefore, we hypothesized that wogonin can induce apoptosis in the HL-60 leukemia cell line. In the present study, the HL-60 cells were treated with different doses of wogonin (0-150 $\mu \mathrm{M})$. Wogonin inhibited the viability of HL-60 cells in a dose-dependent and time-dependent manner. Flow cytometry and analyses of caspase and PARP-1 activation and the $\mathrm{Bax} / \mathrm{Bcl}-2$ ratio, demonstrated that the cytotoxic effect of wogonin on HL-60 cells was mediated by caspase-dependent and mitochondrialdependent apoptosis. Wogonin also induced the expression of certain members of the endoplasmic reticulum (ER) stress pathway (CHOP, GRP94 and GRP78) and the activation of multiple branches of ER stress transducers (IRE1 $\alpha$, PERKeIF $2 \alpha$ and ATF6) in the HL-60 cells. In addition, wogonin reduced the phosphorylation of PI3K and AKT in the HL-60 cells. Furthermore, constitutive activation of AKT induced by adenoviral vectors inhibited the pro-apoptotic effects and ER stress induced by wogonin in the HL-60 cells. In summary, our results indicated that wogonin induced apoptosis and ER stress in HL-60 cells, which was mediated by the inhibition of the PI3K-AKT signaling pathway.
\end{abstract}

Correspondence to: $\mathrm{Dr}$ Chengjun $\mathrm{Hu}$ or Professor $\mathrm{Xin} \mathrm{Xu}$, Department of Hematology, The Affiliated Jiangyin Hospital of Southeast University Medical College, Jiangyin, Jiangsu 214400, P.R. China

E-mail: huchengjun85@163.com

E-mail: xuxinjiangyin@163.com

*Contributed equally

Key words: wogonin, HL-60 cells, apoptosis, ER stress, PI3K-AKT

\section{Introduction}

Leukemia is a common cancer that affects a significant segment of the population, particularly children (1). A recent study by the American Cancer Society (ACS) showed that 47,150 new cases of leukemia were diagnosed in the United States in 2012, whereas 23,540 adults and children died of leukemia during 2012 (2). In clinical therapy, the major strategy for patients with leukemia include bone marrow transplantation, radiotherapy and chemotherapy $(3,4)$. However, the cure rate is low and the side-effects are debilitating; thus, the search for new agents for leukemia patients is urgent.

Apoptosis is the major method by which anticancer agents eliminate cancer cells (5). Apoptosis is controlled by extrinsic and intrinsic pathways (6). The extrinsic pathway involves the death receptor, in which the death domains target caspase- 8 when combined with their corresponding ligands. The activation of caspase- 8 then activates caspase- 3 to ultimately induce apoptosis. The intrinsic pathway of apoptosis is associated with DNA damage. Oligomerization of Bax or Bak promotes the release of mitochondrial cytochrome $c$ into the cytoplasm. Cytochrome $c$ combines with the caspase-9 precursor to form an apoptosis complex. This activation of caspase-9 then activates caspase-3 and poly(ADP-ribose) polymerase (PARP) to induce apoptosis (7). The mitochondrial depolarization and activation of caspase family proteases are the central steps in the process of apoptosis (8), and their associated signaling pathways include intrinsic (mitochondrial-dependent) and endoplasmic reticulum (ER) stress signals $(9,10)$. ER stress occurs when ER homeostasis is lost due to an overload of protein folding in the ER (11). ER stress triggers an evolutionarily conserved response termed the unfolded protein response (UPR) (12). The UPR alters transcriptional and translational programs to cope with the accumulation of unfolded or misfolded proteins. Failure to resolve a proteinfolding defect and restore ER homeostasis induces the UPR to initiate apoptosis. Several mechanisms have been proposed that link the distressed ER to apoptosis, including Bcl-2 family proteins $(13,14)$.

Numerous phytochemicals are present in many herbalbased dietary supplements or herbal medicines, which may be effective in clinical application as cancer suppres- 
sors $(15,16)$. Wogonin is one of the major flavonoids found in the root of the Chinese herb Scutellaria baicalensis Georgi (also called Huang-Qin), which is widely used in the treatment of a number of diseases due to its antiviral, antibacterial, anti-inflammatory, antioxidant and anticancer effects $(17,18)$. Previous studies both in vitro and in vivo have shown that wogonin has anticancer effects in various types of cancer, such as human colorectal (19) and lung (20) cancer, gallbladder carcinoma (21), breast cancer (22), hepatocellular carcinoma (23), osteosarcoma (24) and glioma $(25,26)$. Recent studies have also shown that wogonin exhibits anticancer effects in hematologic malignancies. For example, wogonin induced apoptosis in a human myeloma cell line by downregulating p-AKT and overexpressing Bax (27). Wogonin also induced cell cycle arrest and erythroid differentiation in the imatinib-resistant K562 leukemia cell line and primary chronic myelogenous leukemia cells (28). In addition, wogonin reversed the multidrug resistance of human K562/A02 myelogenous leukemia cells via inhibition of the Nrf2/ARE signaling pathway (29). Moreover, wogonin attenuated etoposide-induced oxidative DNA damage and apoptosis in the bone marrow cells of mice via suppression of oxidative DNA stress and modulation of OGG1 expression (30). These findings indicated that wogonin is a promising chemo-protective agent and may be valuable for the treatment of leukemia. However, the precise mechanisms involved in the induced apoptosis of leukemia cells by wogonin remain to be further elucidated.

The aim of the present study was to investigate the effects of wogonin on the apoptosis of HL- 60 cells. The apoptotic mechanisms and pathways induced by wogonin were also investigated, with particular focus on caspase-, mitochondrialand ER stress-dependent apoptosis. We further examined whether wogonin induces HL-60 cell apoptosis through the PI3K-AKT signaling pathway. Our results support the potential of wogonin as a chemotherapeutic agent for the treatment of leukemia.

\section{Materials and methods}

Reagents and antibodies. Wogonin was purchased from Sigma-Aldrich (St. Louis, MO, USA) and dissolved in dimethylsulfoxide (DMSO; Sigma-Aldrich) as a stock solution of $100 \mathrm{mM}$. Wogonin was further diluted in RPMI-1640 (Invitrogen, Big Cabin, OK, USA) plus 10\% fetal bovine serum (FBS; Invitrogen, Grand Island, NY, USA) to the appropriate final concentrations. The primary polyclonal rabbit anti-human antibodies: PI3K, phosphorylated (p)-PI3K (Tyr458), AKT, p-AKT (Ser473), PARP-1, pancreatic ER stress kinase (PERK), eukaryotic initiation factor $2 \alpha$ (eIF2 $\alpha$ ), activating transcription factor 6 (ATF6), inositol-requiring enzyme $1 \alpha($ IRE $1 \alpha)$ and $\beta$-actin were obtained from Cell Signaling Technology (Beverly, MA, USA). The secondary horseradish peroxidase (HRP)-labeled mouse anti-rabbit IgG polyclonal antibodies for western blot analysis were provided by Merck Millipore (Beijing, China). Annexin V-fluorescein isothiocyanate (FITC) and propidium iodide (PI) were purchased from BD Biosciences (Palo Alto, CA, USA), SH-6 was provided by Santa Cruz Biotechnology (Dallas, TX, USA).
Construction of the AKT constructs. The adenoviral constructs were generated using a protocol from Qbiogene's AdEasy Vector system (Carlsbad, CA, USA) with the following modifications. The constitutively active AKT constructs were amplified by PCR and cloned into the pShuttle-CMV adenovirus transfer vector (BD Clontech Laboratories, Palo Alto, CA, USA). HA-AKT was cloned into the HindIII and EcoRV sites and was transformed into BJ5183 bacteria, resulting in Ad-HA-AKT. All constructs were purified using Qiagen Plasmid Maxiprep kits according to the manufacturer's instructions. The constructs were confirmed by restriction enzyme digestion and sequencing analysis.

The adenoviruses were amplified by infecting human acute promyelocytic leukemia HL-60 (ATCC ${ }^{\circledR}$ CCL-240 ${ }^{\mathrm{TM}}$ ) cells. Infected cells were harvested and centrifuged at $1,250 \mathrm{x} \mathrm{g}$ at $4^{\circ} \mathrm{C}$ for $10 \mathrm{~min}$, and the resulting cellular lysate pellet was resuspended in Dulbecco's modified Eagle's medium (DMEM) without supplements. The cells were disrupted using three freeze/thaw cycles and the suspension was subsequently spun at $1,250 \mathrm{x} \mathrm{g}$ at $4^{\circ} \mathrm{C}$ for $15 \mathrm{~min}$ to release the virus particles. The supernatant containing the viral particles was spun at $100,000 \mathrm{x} \mathrm{g}$ (Beckman SW28 rotor) for $16 \mathrm{~h}$ at $4^{\circ} \mathrm{C}$ through an isopycnic $\mathrm{CsCl}$ gradient. The viral band was isolated and dialyzed against $10 \%$ glycerol in phosphate-buffered saline (PBS; $137 \mathrm{mM} \mathrm{NaCl}, 2.7 \mathrm{mM} \mathrm{KCl}, 1.47 \mathrm{mM} \mathrm{KH}_{2} \mathrm{PO}_{4}$ and $8.1 \mathrm{mM} \mathrm{Na}_{2} \mathrm{HPO}_{4}, \mathrm{pH} \mathrm{7.6)}$ for $16-24 \mathrm{~h}$ at $4^{\circ} \mathrm{C}$. The viral particle concentration (titer) was determined by absorbance at $260 \mathrm{~nm}$. Viruses were aliquoted and stored at $-80^{\circ} \mathrm{C}$ until used.

Cell culture and treatments. The HL-60 suspension cells were purchased from the Peking Union Medical College Cell Library (Beijing, China). The cells were cultured in RPMI-1640 supplemented with $100 \mathrm{U} / \mathrm{ml}$ of penicillin, $100 \mu \mathrm{g}$ streptomycin and $10 \% \mathrm{FBS}$ at $37^{\circ} \mathrm{C}$ in a humidified atmosphere containing $5 \% \mathrm{CO}_{2}$. The media were changed every 2-3 days and subcultured when the cell population density reached $70-80 \%$ confluency. Cells were seeded at an appropriate density according to each experimental design.

Assays of caspase-3, 8 and 9 activities. Cell lysates (30 $\mu \mathrm{g})$ from the HL-60 cells obtained after treatment with wogonin at the desired doses and time periods, were analyzed for caspase acivity spectrophotometrically at $405 \mathrm{~nm}$ using a microtiter plate reader. The assays were performed by incubating the cell lysates with $0.2 \mathrm{mM}$ of the caspase-specific colorimetric tetrapeptide substrates, Ac-LEHD-p-nitroaniline (pNA) (for caspase-9), Ac-IETD-pNA (for caspase-8) or Ac-DEVD-pNA (for caspase-3) for $1 \mathrm{~h}$ at $37^{\circ} \mathrm{C}$ as described in a previous study (31). The increase in the absorbance at $405 \mathrm{~nm}$ which corresponds to the amount of pNA liberated from the peptide substrates was converted into units of enzyme activity using a standard curve generated with free pNA. One unit of caspase-3, 8 or 9 activity corresponded to the amount of enzyme that will release 1 pmol of pNA from $0.2 \mathrm{mM}$ DEVD-pNA, IETD-pNA or Ac-LEHD-pNA/min, respectively. Lysates from the HL-60 cells treated with DMSO were also used in these assays as the control group.

Cell viability assay. Cell viability was assessed using the MTT assay. HL-60 cells were plated into 96-well clusters at 
a density of $5 \times 10^{4}$ cells/well. After a $24-\mathrm{h}$ incubation under group-specified experimental conditions, the clustered HL-60 cells were processed for detection of cell viability by MTT assays. Spent medium was removed and $10 \mu \mathrm{l}$ MTT solution ( $5 \mathrm{mg} / \mathrm{ml}$ ) was added to $100 \mu \mathrm{l}$ of respective growth medium without phenol red, and the plates were incubated at $37^{\circ} \mathrm{C}$ for $4 \mathrm{~h}$ in a humidified $5 \% \mathrm{CO}_{2}$ atmosphere. Then, the formazan crystals formed by mitochondrial reduction of MTT were solubilized in DMSO (100 $\mu \mathrm{l} /$ well), and the absorbance was read at $540 \mathrm{~nm}$ using a microplate reader (Bio-Rad, Hercules, CA, USA). Percent inhibition of cytotoxicity was calculated as a fraction of the control group and expressed as a percentage of cell viability.

Determination of apoptosis in the HL-60 cells. Doublestaining for Annexin V-FITC and PI was performed to estimate the apoptotic rate of the HL-60 cells. Briefly, after incubation with various doses of wogonin for $48 \mathrm{~h}$, the HL-60 cells were trypsinized and washed twice with PBS, and centrifuged at $800 \mathrm{rpm}$ for $5 \mathrm{~min}$. Then, $1 \times 10^{6}$ cells were suspended in binding buffer and double-stained with Annexin V-FITC and PI for $30 \mathrm{~min}$ at room temperature. Subsequently, the fluorescence of each sample was quantitatively analyzed by a FACSCalibur flow cytometer and CellQuest software. The results were interpreted as follows: PI-positive and Annexin V-FITC-positive stained cells were considered as apoptotic.

Quantitative reverse transcriptase-polymerase chain reaction ( $q R T-P C R)$. Total messenger RNA from the HL-60 cells was isolated using the RNeasy Mini kit (Qiagen, Valencia, CA, USA) according to the manufacturer's instructions. The amount and quality of the RNA were determined using a NanoDrop 2000 spectrophotometer (Thermo Scientific, Wilmington, DE, USA). First-strand cDNA was synthesized from $300 \mathrm{ng}$ of RNA with SuperScript ${ }^{\circledR}$ III First-Strand Synthesis system (Applied Biosystems, Foster City, CA, USA) and used as the template for PCR with the FastStart HiFi PCR system (Roche Applied Science, Indianapolis, IN, USA). The fold-change in gene expression was obtained by dividing the treated group signal by that of the base expression level signal of the corresponding gene in the untreated cells. Results were normalized using qRT-PCR signal from $\beta$-actin of the respective samples.

The primers of the target genes were: glucose-regulated protein 78 (GRP78) forward, 5'-TCT GCT TGA TGT GTG TCC TCT T-3' and reverse, 5'-GTC GTT CAC CTT CGT AGA CCT-3'; C/EBP-homologous protein (CHOP) forward, 5'-GGA GAA GGA GCA GGA GAA TGA-3' and reverse, 5'-AGA CAG ACA GGA GGT GAT GC-3'; GRP94 forward, 5'-TCG GGA AGC AAC AGA GAA GG-3' and reverse, 5'-TCA TCT TCC TTA ACC CTC CGC-3'; Bcl-2 forward, 5'-GCT GAG GCA GAA GGG TTA TG-3' and reverse, 5'-GCC CCC TTG AAA AAG TTC AT-3'; Bax forward, 5'-AGG GTT TCA TCC AGG ATC GAG CAG-3' and reverse, 5'-ATC TTC TTC CAG ATG GTG AGC GAG-3'; $\beta$-actin forward, 5'-GGC GGA CTA TGA CTT AGT TG-3' and reverse, 5'-AAA CAA CAA TGT GCA ATC AA-3'.

Western blot assay. For the western blot analysis, HL-60 cells were harvested, washed once in ice-cold PBS, gently lysed in ice-cold lysis buffer $(250 \mathrm{mM}$ sucrose, $1 \mathrm{mM}$ EDTA, $0.05 \%$ digitonin, $25 \mathrm{mM}$ Tris, $\mathrm{pH} 6.8,1 \mathrm{mM}$ dithiothreitol, $1 \mu \mathrm{g} / \mathrm{ml}$ leupeptin, $1 \mu \mathrm{g} / \mathrm{ml}$ pepstatin, $1 \mu \mathrm{g} / \mathrm{ml}$ aprotinin, $1 \mathrm{mM}$ benzamidine and $0.1 \mathrm{mM}$ phenylmethylsulphonyl fluoride) for $30 \mathrm{~min}$, and centrifuged at $12,000 \mathrm{rpm}$ at $4^{\circ} \mathrm{C}$. The protein concentration was measured using the BioRad Bradford protein assay reagent, and subjected to SDS-PAGE. Proteins were transferred to polyvinylidene fluoride membranes and incubated successively in 5\% bovine serum albumin in Tris-

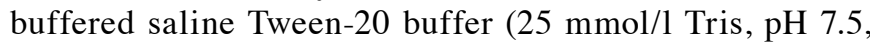
$150 \mathrm{mmol} / 1 \mathrm{NaCl}$ and $0.1 \%$ Tween-20) for $1 \mathrm{~h}$, then incubated overnight at $4^{\circ} \mathrm{C}$ with PI3K, p-PI3K (Tyr458), AKT, p-AKT (Ser473), PARP-1, PERK, eIF2 $\alpha$, ATF6, IRE1 $\alpha$ or $\beta$-actin, followed by reaction with HRP-labeled mouse anti-rabbit IgG polyclonal antibodies for $1 \mathrm{~h}$. After each incubation, the membranes were washed extensively in TTBS and the immunoreactive band was detected using ECL-detecting reagents.

Data analysis. Results are expressed as the mean \pm SEM. Statistical comparisons were performed using a Student's t-test, one-way analysis of variance (ANOVA), or two-way repeated measures ANOVA with the Student's t-test for post hoc analyses. In all cases, values of $\mathrm{P}<0.05$ were considered to indicate statistically significant results.

\section{Results}

Cytotoxic effects of wogonin on the HL-60 cells. In the pre-experiment, we applied wogonin at doses of 10, 25, $50,75,100$ and $150 \mu \mathrm{M}$. The results showed that wogonin inhibited the viability of HL-60 cells in a dose-dependent manner (Fig. 1a) and the effect was predominant at 75-150 $\mu \mathrm{M}$. In addition, HL-60 cells were treated with $75 \mu \mathrm{M}$ wogonin for $6,12,24,48,72$ and $96 \mathrm{~h}$. We found that $75 \mu \mathrm{M}$ wogonin inhibited the viability of the HL-60 cells in a time-dependent manner (Fig. 1b) and the effect was predominant at 48-96 h. Therefore, HL-60 cells were treated with wogonin for $48 \mathrm{~h}$ in the subsequent experiments.

Wogonin induces the apoptosis of HL-60 cells. To further investigate whether wogonin induces the apoptosis of HL-60 cells, the cells were treated with $75 \mu \mathrm{M}$ wogonin for $48 \mathrm{~h}$ and the apoptotic rate of the HL-60 cells was detected using Annexin V-FITC/PI staining. The results revealed that $75 \mu \mathrm{M}$ wogonin induced apoptosis in the HL-60 cells (Fig. 1c).

Effects of wogonin on the caspase activity of HL-60 cells. Apoptosis induced by various cytotoxic agents is highly dependent on the activation of caspases, which play pivotal roles in cleaving specific target proteins (32). As shown in Fig. 1c, wogonin caused strong apoptotic death of the HL-60 cells. Therefore, we assessed whether wogonin activates caspase pathways in the HL-60 cells. Cells were exposed to increasing doses of wogonin for $48 \mathrm{~h}$, and then analyzed for caspase activation by colorimetric assays. As shown in Fig. 2a-c, wogonin triggered a dose-dependent increase in the specific activities of caspase-3, -8 and -9 . A mean specific activity peak was noted after incubation of the HL-60 cells with doses of 75-150 $\mu \mathrm{M}$ wogonin for $48 \mathrm{~h}$. 

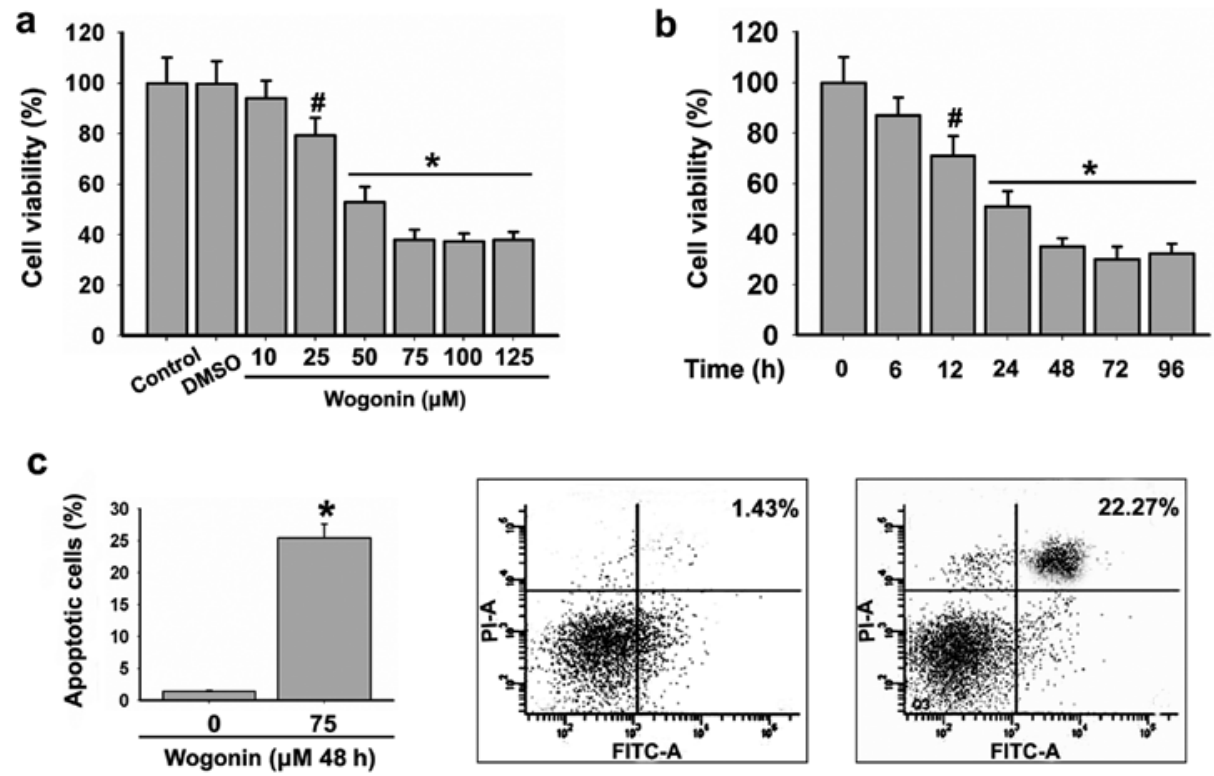

Figure 1. Effect of wogonin on cell viability and apoptosis in HL-60 cells. Cell viability was assayed by the MTT method. (a) Effect of different doses of wogonin on the cell viability of HL-60 cells. The effect was dose-dependent. (b) Effect of $75 \mu \mathrm{M}$ wogonin on the cell viability of HL-60 cells for different time periods. The effect was time-dependent manner. The control group was set at $100 \%$. (c) Cell apoptosis was determined by the Annexin V/PI flow cytometric assay method, which detects cells in an early stage of the apoptotic pathway and distinguishes among apoptotic and necrotic cells. Treatment with $75 \mu \mathrm{M}$ wogonin induced the apoptosis of HL-60 cells. All data are presented as means $\pm \mathrm{SD}$ ( $\mathrm{n}=6,{ }^{\prime \prime} \mathrm{P}<0.05,{ }^{*} \mathrm{P}<0.01$ significantly different from the control group).

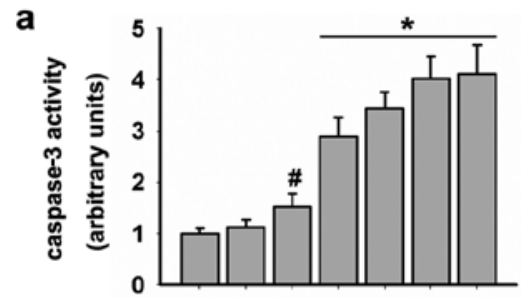

Wogonin $(\mu \mathrm{M}) \quad 0 \quad 1025 \quad 50 \quad 75100150$ b

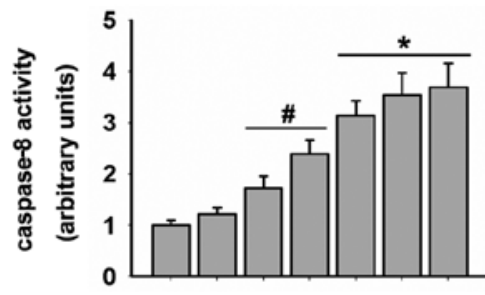

Wogonin $(\mu \mathrm{M}) \quad 0 \quad 10 \quad 25 \quad 50 \quad 75100150$

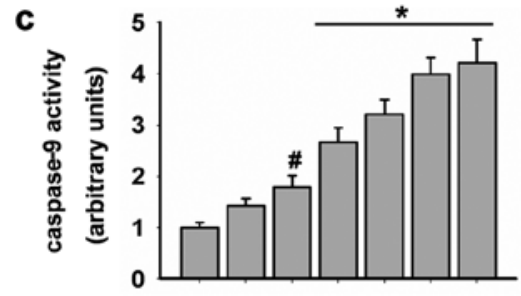

Wogonin $(\mu \mathrm{M}) \quad 0 \quad 10 \quad 2550 \quad 75100150$
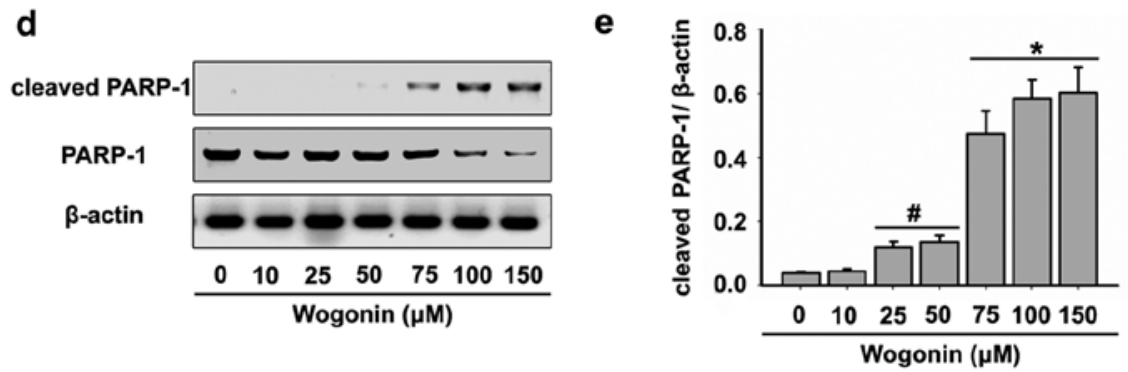

f

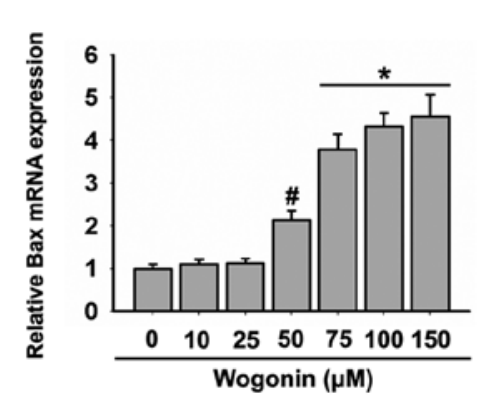

g

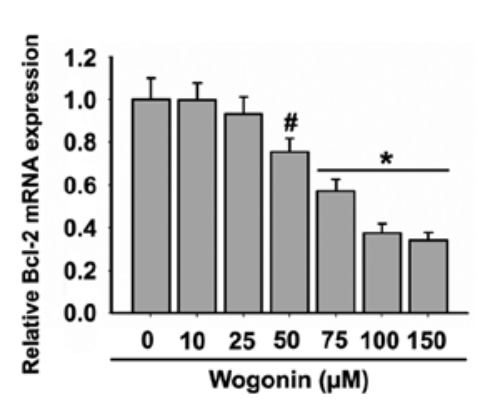

h

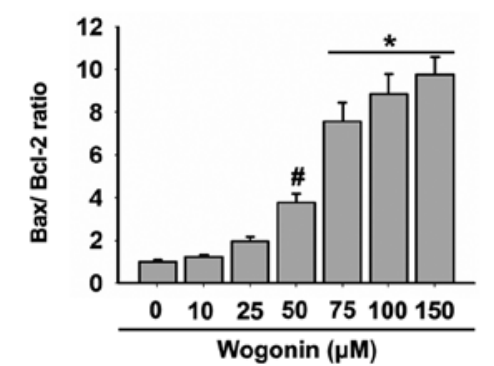

Figure 2. Effect of wogonin on the activation of caspase- and mitochondrial-dependent events in HL-60 cells. Activation of (a) caspase-3, (b) caspase-8 and (c) caspase- 9 was measured in HL-60 cells following treatment with various concentrations of wogonin. The control group was set at 1 . Activation of (d) PARP-1 was measured in the HL-60 cells following treatment with various concentrations of wogonin by western blot assay. (e) The histogram shows the ratio of cleaved PARP-1 and total PARP-1 to $\beta$-actin in the HL-60 cells. The expression levels of the (f) Bax and ( $\mathrm{g}$ ) Bcl-2 genes were measured in HL-60 cells by qRT-PCR. The control group was set at 1 . (h) Ratio of Bax/Bcl-2 mRNA levels after wogonin treatment in the HL-60 cells. Data are the mean \pm SEM ( $\mathrm{n}=6$, ${ }^{\#} \mathrm{P}<0.05,{ }^{*} \mathrm{P}<0.01$ significantly different from the control group). 

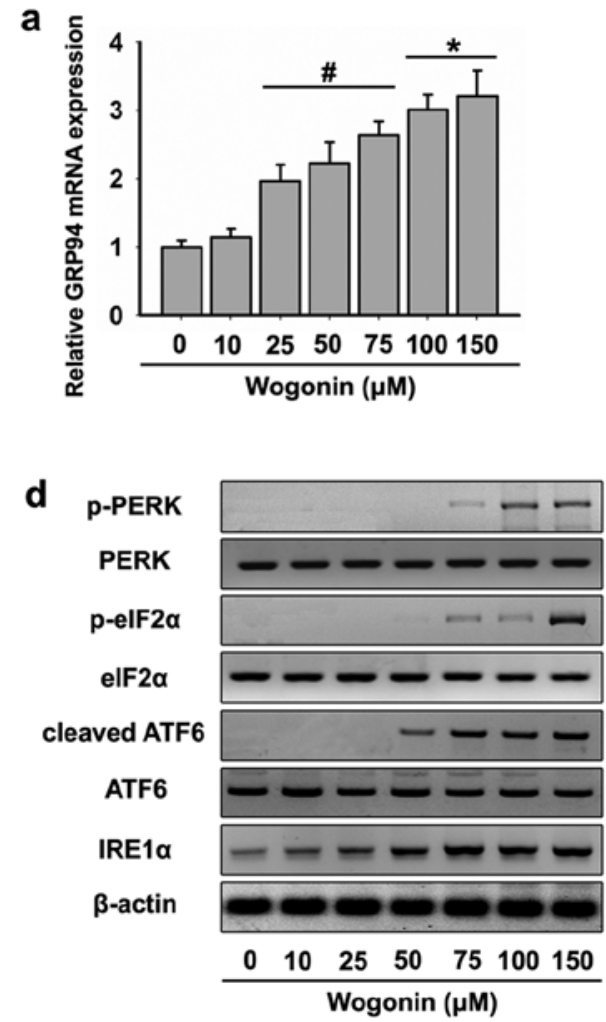

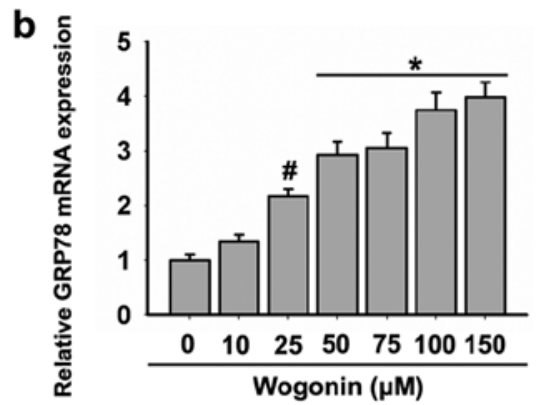

e

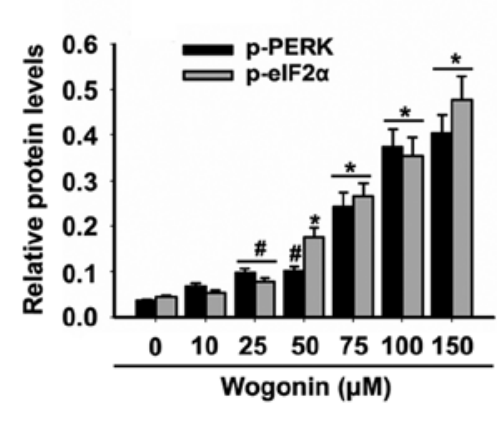

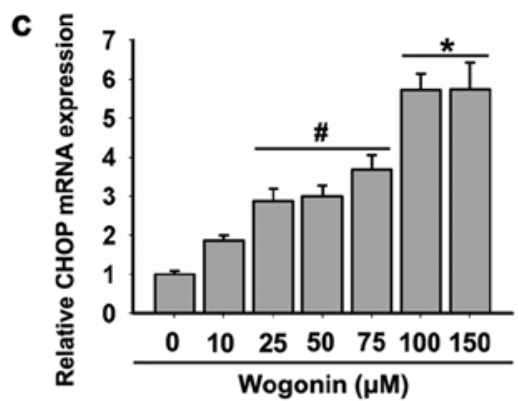

f

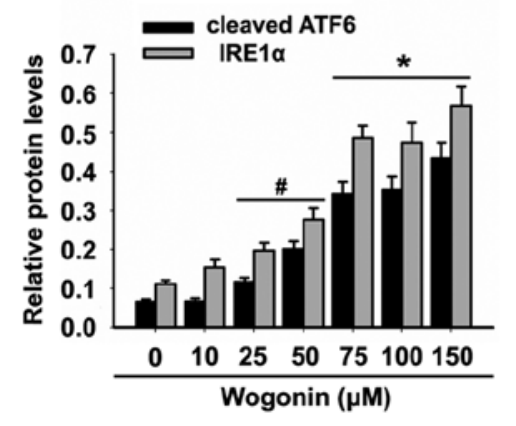

Figure 3. Effects of wogonin on ER stress in HL-60 cells. The expression levels of (a) GRP94, (b) GRP78 and (c) CHOP genes were measured in HL-60 cells following treatment with various doses of wogonin by qRT-PCR. The control group was set at 1 . (d) The expression levels of p-PERK, p-eIF2 $\alpha$, cleaved ATF6 and IRE1 $\alpha$ were measured in HL-60 cells following treatment with increasing doses of wogonin by western blot assay. (e) The histogram shows the ratio of p-PERK and p-eIF2 $\alpha$ to $\beta$-actin for each dose of wogonin in the HL-60 cells. (f) The histogram shows the ratio of cleaved ATF6 and IRE1 $\alpha$ to $\beta$-actin for each dose of wogonin in HL-60 cells. These data are mean \pm SEM $\left(n=6,{ }^{,} \mathrm{P}<0.05,{ }^{*} \mathrm{P}<0.01\right.$ significantly different from the control group). $\mathrm{p}$-PERK and $\mathrm{p}$-eIF2 $\alpha$, phosphorylated PERK and eIF2 $\alpha$.

Effects of wogonin on the PARP-1 activity of HL-60 cells. We next performed western blot analysis to determine the level of cleaved PARP-1 that induces the enhanced apoptosis. The cleavage of PARP-1 was significantly higher in the HL-60 cells following wogonin treatment in a dose-dependent manner. The maximum activation of cleaved PARP-1 was detected following treatment of wogonin at doses of 75-150 $\mu \mathrm{M}$ for $48 \mathrm{~h}$ (Fig. $2 \mathrm{~d}$ and e). This result was in accordance with the activities of caspase-3, 8 and 9 . In addition, wogonin treatment also resulted in significant reduction in total PARP-1 levels (Fig. 2d and e).

Effects of wogonin on the mitochondrial death pathway in HL-60 cells. The activation of caspase-9 by wogonin suggested that the mitochondrial apoptotic pathway was triggered in the HL-60 cells. Since Bcl-2 family proteins are known to control the mitochondrial-mediated apoptosis pathway by maintaining a balance between pro- and antiapoptotic members (33), we examined the effects of wogonin on the expression levels of Bcl-2 family proteins in the HL-60 cells. Our results showed that wogonin increased the mRNA level of pro-apoptotic Bax (Fig. 2f), but decreased the mRNA level of Bcl-2 (Fig. 2g) in HL-60 cells. In addition, the Bax/ $\mathrm{Bcl}-2$ ratio was increased following treatment with wogonin in the HL-60 cells (Fig. 2h). These data demonstrated that wogonin induced HL-60 cell apoptosis through mitochondrial-mediated mechanisms.
Effects of wogonin on the ER stress in HL-60 cells. Treatment with wogonin has been shown to induce accumulation of misfolded nascent glycoproteins in the ER lumen in various types of cancer cells, leading to ER stress and UPR activation $(23,24,26,34)$. The expression of ER stress markers was analyzed in the HL-60 cells following treatment with wogonin. In the HL-60 cells, wogonin increased the mRNA expression of GRP94 (Fig. 3a), GRP78 (Fig. 3b) and CHOP (Fig. 3c) confirming induction of ER stress, and their increased expression was correlated with cleaved PARP-1 and activity of caspase- $-3,-8$ and -9 , markers of apoptosis.

When ER stress occurs, GRP78 is released from three key branches: PERK, IRE1 and ATF6 and binds misfolded proteins, thereby activating the UPR $(35,36)$. Next, we analyzed the expression levels of the following key UPR signal transduction molecules: PERK, eIF2 $\alpha$, ATF6 and IRE1 $\alpha$. Exposure of the HL-60 cells to wogonin resulted in the upregulated expression of p-PERK, p-eIF2 $\alpha$ and cleaved ATF6 and activation of IRE1 $\alpha$ (Fig. 3d-f). These data suggested that wogonin induced HL-60 cell apoptosis through ER stress-mediated mechanisms.

Effects of wogonin on the PI3K-AKT activation in HL-60 cells. To further understand the molecular mechanisms by which wogonin induces HL-60 cell apoptosis, we examined the expression of PI3K-AKT, critical signaling proteins associated with cell apoptosis. The levels of p-PI3K and p-AKT 

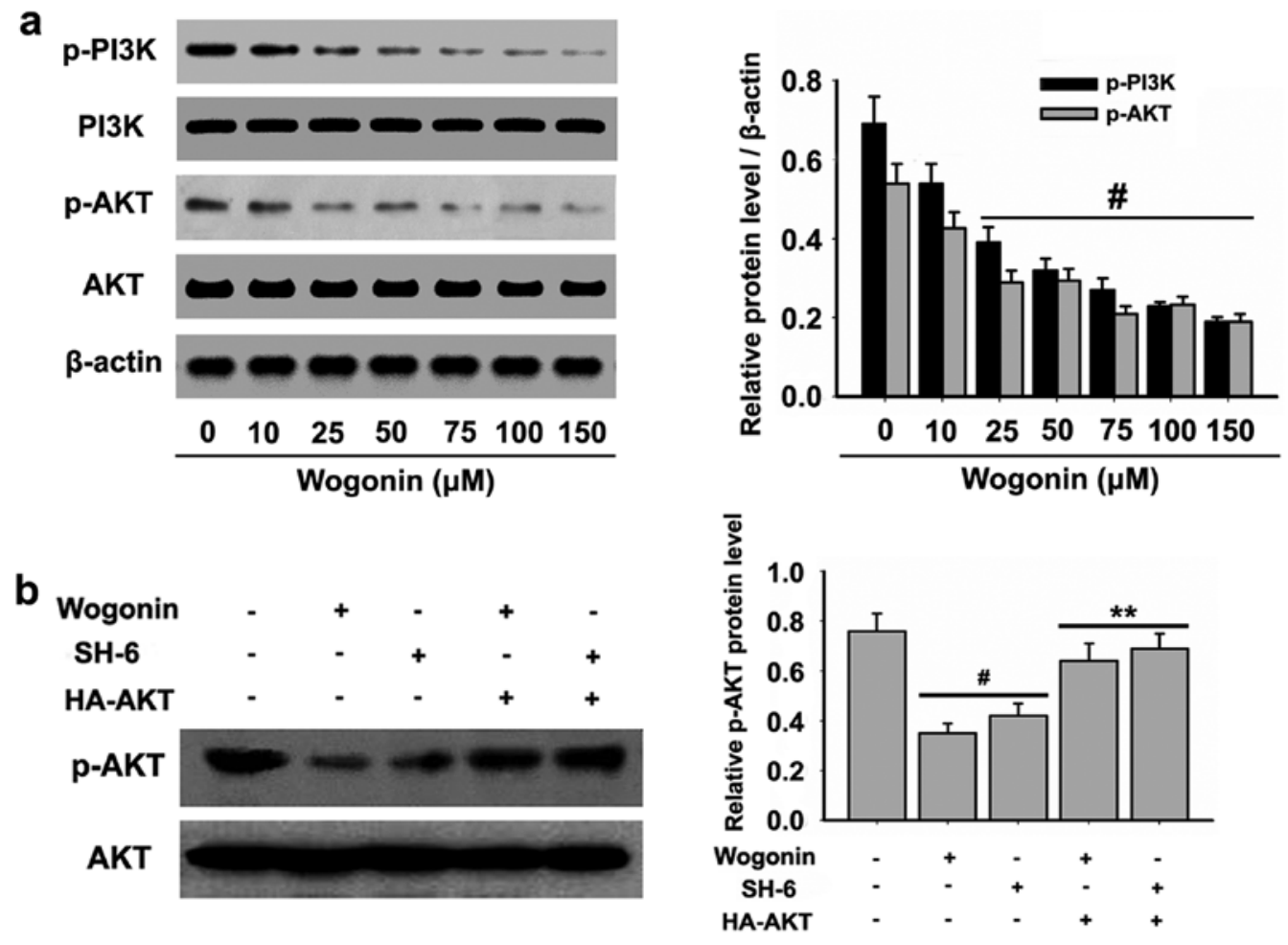

Figure 4. Effect of wogonin on the PI3K/AKT signaling pathway in HL-60 cells. (a) The expression levels of p-PI3K and p-AKT were measured in the HL-60 cells following treatment with wogonin at various doses by western blot assay. The histogram shows the ratio of p-PI3K and p-AKT to $\beta$-actin for each dose of wogonin in the HL-60 cells. (b) The expression level of p-AKT was measured in the HL- 60 cells following treatment with $75 \mu$ M wogonin, SH- 6 and/ or activation of AKT induced by adenoviral vectors by western blot assay. The selective AKT inhibitor SH-60 was used as the negative control. The histogram shows the ratio of $\mathrm{p}$-AKT to $\beta$-actin for each group in the HL-60 cells. These data are means $\pm \mathrm{SEM}\left(\mathrm{n}=6\right.$, ${ }^{\prime} \mathrm{P}<0.01$, significantly different from the control group; ${ }^{* *} \mathrm{P}<0.01$ significantly different from the wogonin only treatment group).

were detected, and the results demonstrated that wogonin significantly blocked the constitutive phosphorylation of PI3K at Tyr458 and AKT at Ser473 in a dose-dependent manner (Fig. 4a).

To determine the role of PI3K-AKT in wogonin-induced apoptosis and ER stress in HL-60 cells, constitutively active HA-tagged AKT constructs were made in AKT at Ser473, which were mutated to an aspartic acid residue to mimic phosphorylated AKT. The expression of HA-AKT significantly increased the phosphorylated AKT activity (Fig. 4b) after wogonin treatment. Active AKT significantly increased the cell viability (Fig. 5a) and reduced the percentage of HL-60 apoptotic cells (Fig. 5b) when compared with the cells in the wogonin only treatment group. Moreover, active AKT also reduced wogonin-induced caspase-3, -8, -9 (Fig. 5c) and PARP-1 (Fig. 5d) activities and the Bax/Bcl-2 ratio (Fig. 5e-g) when compared with cells in the wogonin only treatment group. In addition, upregulated expression of p-PERK, p-eIF $2 \alpha$ and cleaved ATF6 and activation of IRE1 $\alpha$ induced by wogonin were reduced in the HL- 60 cells expressing active AKT (Fig. 6a-c). These results indicated that the pro-apoptotic effects of wogonin in HL-60 cells were associated with inhibition of the activation of the PI3K/AKT signaling cascade.

\section{Discussion}

Wogonin, one of the active components extracted from Scutellariae radix, exhibits antioxidant, anti-inflammatory and antitumor activities. Wogonin has been shown to induce anti-proliferation, cell cycle arrest and differentiation in hematologic malignancies. Our study presented data showing that wogonin induced a cytotoxic effect and apoptosis in HL-60 cells. Activation of caspase- 8 and -9 are key events in the extrinsic and intrinsic pathways of apoptosis, respectively, while the activation of caspase-3 and PARP-1 is a critical event in both apoptotic pathways. Accordingly, caspases-3, -8, -9 and PARP-1 are the main markers for the activation of apoptosis $(6,7)$. Furthermore, the effect of wogonin on the activation of caspase- $3,-8,-9$ and PARP-1 was investigated to attain the precise mechanism for the induction of apoptosis by wogonin in HL-60 cells. We found that the induction in activation of caspase- $3,-8,-9$ and PARP-1 by wogonin might be a reason for the pro-apoptotic effects in HL-60 cells.

Previous studies have shown that wogonin triggers the apoptosis of human osteosarcoma (25), hepatocellular carcinoma (23) and glioma cancer cells (26) through the ER stress-dependent signaling pathways. Moreover, wogonin elicits a potent antitumor immune effect in human and mouse gastric carcinoma cells through an ER stress/AKT dependent manner (34). In addition, the apoptosis of cancer cells can be induced via ER stress. ER stress occurs when ER homeostasis is lost due to an overload of protein folding in the ER (11). ER stress triggers an evolutionarily conserved response termed the UPR (37). The UPR is mediated by three ER-resident transmembrane proteins that sense ER stress and signal downstream pathways. These proximal sensors 

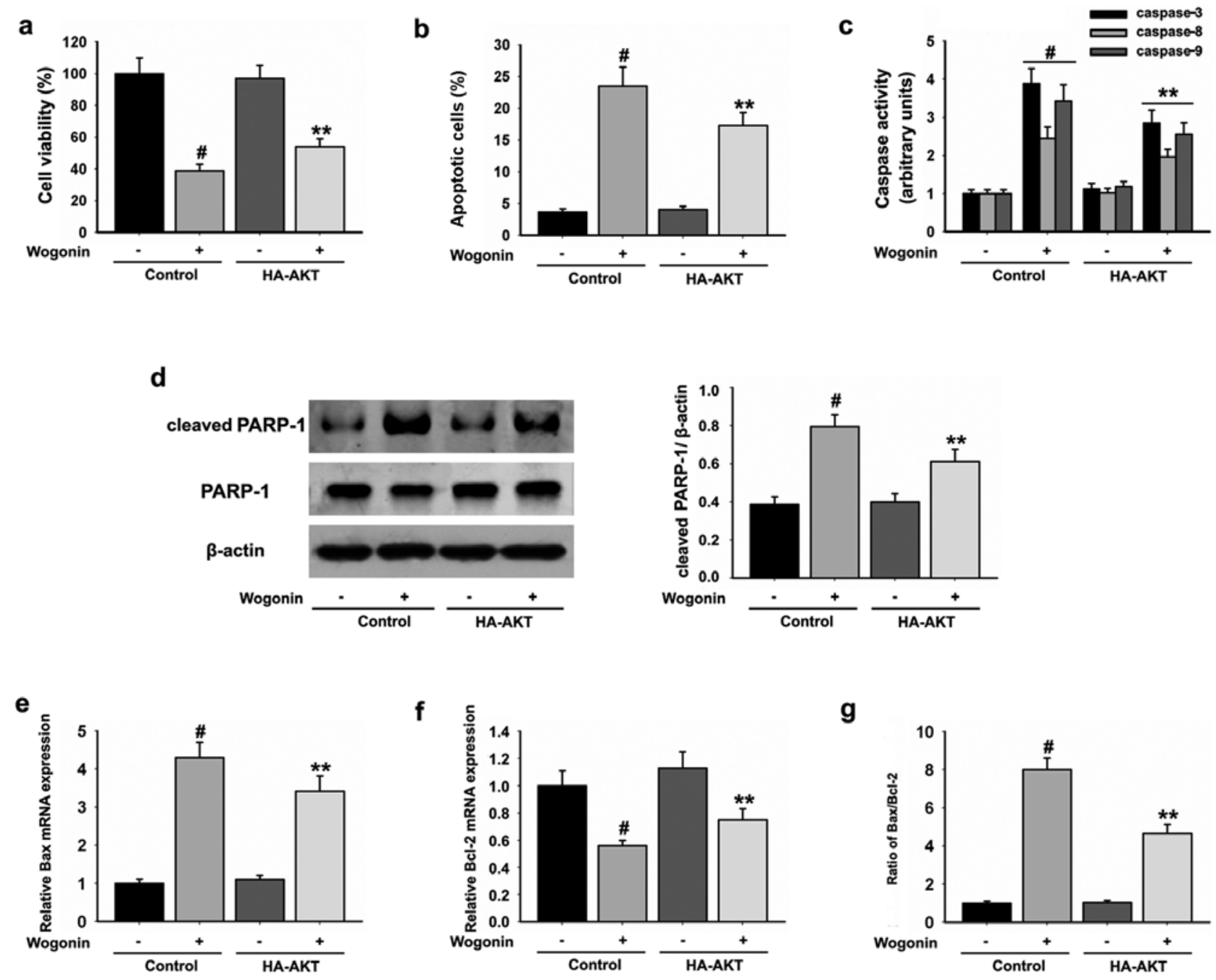

Figure 5. Activation of the PI3K/AKT signaling pathway inhibits the pro-apoptotic effect of wogonin in HL-60 cells. Cell viability was assayed by the MTT method. (a) Effect of active AKT on the cell viability of the HL-60 cells after $75 \mu \mathrm{M}$ wogonin treatment is shown. Cell apoptosis of HL-60 cells was determined by the Annexin V/PI flow cytometric assay method. (b) Effect of active AKT on the level of apoptosis in the HL-60 cells after $75 \mu \mathrm{M}$ wogonin treatment is shown. (c) Effect of active AKT on the activation of caspase-3, -8 and -9 was measured in HL-60 cells after $75 \mu$ M wogonin treatment by western blot assay. (d) Effect of active AKT on the activation of PARP-1 was measured in HL-60 cells after $75 \mu \mathrm{M}$ wogonin treatment by western blot assay. The histogram shows the ratio of cleaved PARP-1 and total PARP-1 to $\beta$-actin in the HL-60 cells. (e) Effect of active AKT on the expression level of the BAX gene was measured in HL-60 cells after $75 \mu \mathrm{M}$ wogonin treatment by qRT-PCR. (f) Effect of active AKT on the expression level of Bcl-2 gene was measured in HL-60 cells after $75 \mu \mathrm{M}$ wogonin treatment by qRT-PCR. (g) Effect of active AKT on the ratio of Bax/Bcl-2 mRNA after $75 \mu \mathrm{M}$ wogonin treatment in the HL-60 cells. These data are means \pm SEM $\left(n=6,{ }^{*} \mathrm{P}<0.01\right.$, significantly different from the control group; ${ }^{* *} \mathrm{P}<0.01$ significantly different from the wogonin only treatment group).

include IRE1 $\alpha$, PERK and ATF6 (38). Here, we observed that wogonin upregulated the expression of p-PERK, p-eIF2 $\alpha$, cleaved ATF6 and activation of IRE1 $\alpha$ in a dose-dependent manner in HL-60 cells. It is well known that GRP78 plays a critical cytoprotective role against ER stress (39-41). In non-stressed mammalian cells, GRP78 constitutively binds to ATF6, PERK and IRE1 and maintains them in an inactive status. Upon ER stress, sequestration of GRP78 by unfolded proteins activates these sensors and initiates the UPR $(39,40)$. The increased mRNA expression levels of GRP78 and GRP94 were detected in HL-60 cells after treatment with wogonin. Following GRP78 and GRP94 induction, ER stress signals lead to activation of CHOP which has been reported to sensitize cells to apoptosis (38-40). In our present study, wogonin treatment was found to elevate expression of CHOP mRNA, which might be another reason for the pro-apoptotic effects noted in the HL-60 cells.
Bcl-2 family proteins also localize upon ER stress where their proposed functions include regulation of apoptosis and the UPR $(13,14)$. The differential effect of the UPR on cell survival or death has been attributed to the levels of pro- or anti-apoptotic Bcl-2 family members upon ER stress $(13,14)$. Anti-apoptotic Bcl-2 family members possess a hydrophobic groove that binds and inhibits their pro-apoptotic counterparts, which forms the basis of resistance to chemotherapy (42). To overcome this resistance and facilitate cell death, small-molecule inhibitors of the Bcl-2 family, aimed at dislodging the pro-apoptotic members from the hydrophobic groove, have been developed $(43,44)$. In the present study, we demonstrated that wogonin-induced apoptosis in HL-60 cells was associated with increased expression of the pro-apoptotic protein Bax and decreased expression of the anti-apoptotic protein Bcl-2. Wogonin was found to induce an increase in the Bax to Bcl-2 ratio, and increased expression of cleaved caspase- 3 and 9 
a

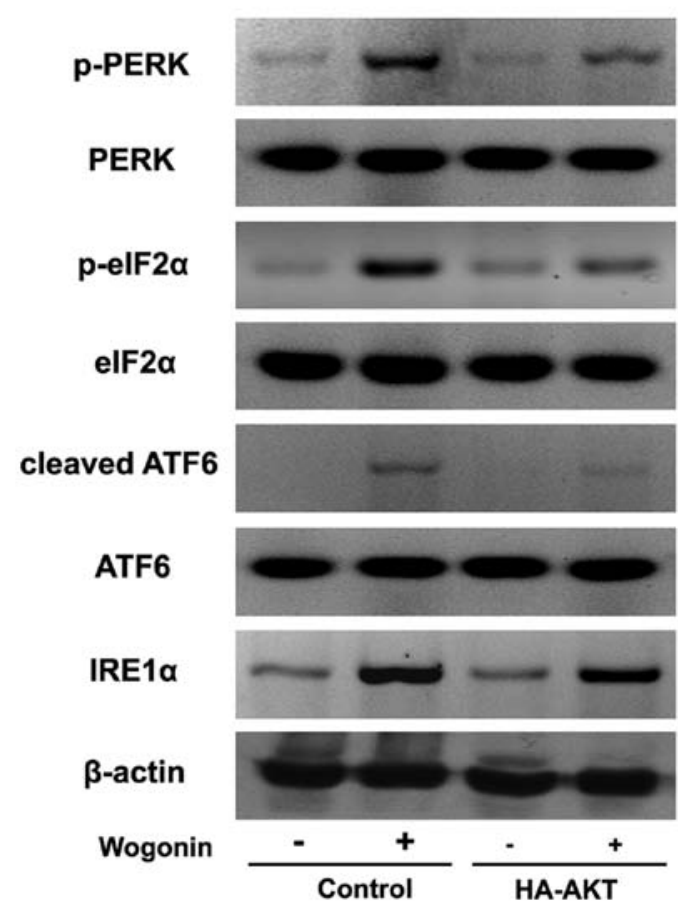

b

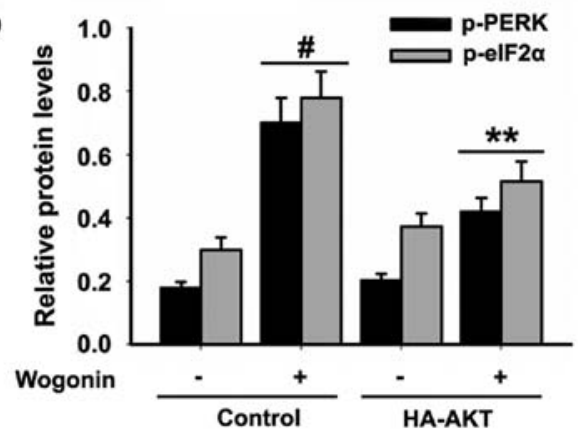

C

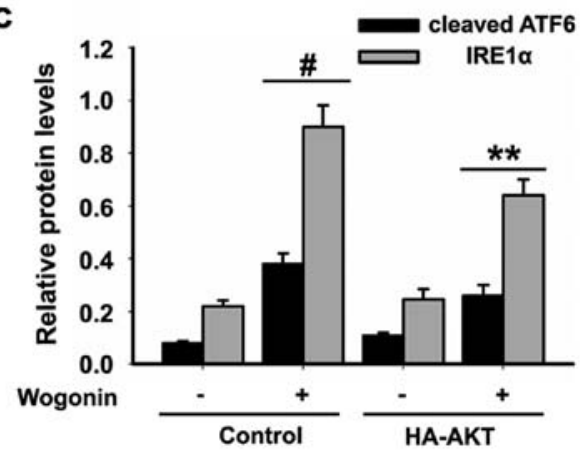

Figure 6. Activation of the PI3K/AKT signaling pathway inhibits the ER stress in wogonin-treated HL-60 cells. (a) Effect of active AKT on the activation of p-PERK, p-eIF2 $\alpha$, cleaved ATF6 and IRE1 $\alpha$ was measured in HL-60 cells after $75 \mu \mathrm{M}$ wogonin treatment by western blot assay. (b) The histogram shows the ratio of p-PERK and p-eIF2 $\alpha$ to $\beta$-actin for each dose of wogonin in the HL-60 cells. (c) The histogram shows the ratio of cleaved ATF6 and IRE1 $\alpha$ to $\beta$-actin for each dose of wogonin in the HL- 60 cells. These data are means \pm SEM $\left(n=6,{ }^{*} \mathrm{P}<0.01\right.$, significantly different from the control group; ${ }^{* *} \mathrm{P}<0.01$ significantly different from the wogonin only treatment group).

has been demonstrated in human breast cancer and myeloma cells $(27,45)$. In the present study, we obtained similar results. Therefore, it is reasonable to conclude that wogonin-induced apoptosis of HL-60 cells is mediated by the mitochondrial pathway through modulation of the Bax to Bcl-2 ratio.

The PI3K/AKT signaling pathway is pivotal in transmitting signals from membrane receptors to downstream targets that regulate critical cellular responses, such as proliferation, apoptosis, differentiation and senescence (46). Previous evidence indicates that the PI3K/AKT signaling pathway plays critical roles in controlling cell survival by suppressing ER stress-induced cell death (47). The important functions of the PI3K/AKT signaling pathway in apoptosis regulation have been extensively studied. Recent studies found that wogonin induced apoptosis via the PI3K/AKT pathway in HT-29 human colorectal cancer cells (48) and in a human myeloma cell line (27). Therefore, we hypothesized that the PI3K/AKT signaling pathway plays various roles in the anti-apoptotic effect of woginin in HL-60 cells. Our data revealed that wogonin downregulated the PI3K/AKT signaling pathway, and activation of AKT induced by adenoviral vectors inhibited the pro-apoptotic effects and ER stress induced by wogonin in the HL-60 cells.

In conclusion, the present study demonstrated that wogonin acted as a strong and selective inducer of apoptosis in the HL-60 cells. Obviously, caspase-, mitochondrial- and ER stress-dependent events are the determinant factors in wogonin-induced cell death. In addition, wogonin downregulated and inactivated the PI3K/AKT signaling pathway, which may have a critical function in wogonin-induced apoptosis in HL-60 cells. Therefore, wogonin is a potential chemotherapeutic agent for the treatment of human leukemia.

\section{References}

1. Canadian Cancer Society/National Cancer Institute of Canada: Canadian Cancer Statistics. ISSN, Toronto, ON, pp0835-2976, 2005.

2. Siegel R, Naishadham D and Jemal A: Cancer statistics, 2013. CA Cancer J Clin 63: 11-30, 2013.

3. Nau KC and Lewis WD: Multiple myeloma: diagnosis and treatment. Am Fam Physician 78: 853-859, 2008.

4. Fotoohi AK, Assaraf YG, Moshfegh A, Hashemi J, Jansen G, Peters GJ, Larsson C and Albertioni F: Gene expression profiling of leukemia T-cells resistant to methotrexate and 7-hydroxymethotrexate reveals alterations that preserve intracellular levels of folate and nucleotide biosynthesis. Biochem Pharmacol 77: 1410-1417, 2009.

5. Kelloff GJ, Crowell JA, Steele VE, Lubet RA, Malone WA, Boone CW, Kopelovich L, Hawk ET, Lieberman R, Lawrence JA, et al: Progress in cancer chemoprevention: development of diet-derived chemopreventive agents. J Nutr 130 (Suppl): 467S-471S, 2000.

6. Chiantore MV, Vannucchi S, Mangino G, Percario ZA, Affabris E, Fiorucci G and Romeo G: Senescence and cell death pathways and their role in cancer therapeutic outcome. Curr Med Chem 16: 287-300, 2009.

7. Meier P and Vousden KH: Lucifer's labyrinth-ten years of path finding in cell death. Mol Cell 28: 746-754, 2007.

8. Lavrik IN, Golks A and Krammer PH: Caspases: pharmacological manipulation of cell death. J Clin Invest 115: 2665-2672, 2005.

9. Kadowaki H, Nishitoh H and Ichijo H: Survival and apoptosis signals in ER stress: The role of protein kinases. J Chem Neuroanat 28: 93-100, 2004. 
10. Oyadomari S and Mori M: Roles of CHOP/GADD153 in endoplasmic reticulum stress. Cell Death Differ 11: 381-389, 2004.

11. Kim I, Xu W and Reed JC: Cell death and endoplasmic reticulum stress: disease relevance and therapeutic opportunities. Nat Rev Drug Discov 7: 1013-1030, 2008.

12. Ron D and Walter P: Signal integration in the endoplasmic reticulum unfolded protein response. Nat Rev Mol Cell Biol 8: 519-529, 2007.

13. Oakes SA, Lin SS and Bassik MC: The control of endoplasmic reticulum-initiated apoptosis by the BCL-2 family of proteins. Curr Mol Med 6: 99-109, 2006.

14. Rong Y and Distelhorst CW: Bcl-2 protein family members: versatile regulators of calcium signaling in cell survival and apoptosis. Annu Rev Physiol 70: 73-91, 2008.

15. Lee KW, Bode AM and Dong Z: Molecular targets of phytochemicals for cancer prevention. Nat Rev Cancer 11: 211-218, 2011.

16. Dumontet $\mathrm{C}$ and Jordan MA: Microtubule-binding agents: a dynamic field of cancer therapeutics. Nat Rev Drug Discov 9: 790-803, 2010

17. Gasiorowski K, Lamer-Zarawska E, Leszek J, Parvathaneni K, Yendluri BB, Błach-Olszewska Z and Aliev G: Flavones from root of Scutellaria baicalensis Georgi: drugs of the future in neurodegeneration? CNS Neurol Disord Drug Targets 10: 184-191, 2011.

18. Li-Weber M: New therapeutic aspects of flavones: the anticancer properties of Scutellaria and its main active constituents Wogonin, Baicalein and Baicalin. Cancer Treat Rev 35: 57-68, 2009.

19. He L, Lu N, Dai Q, Zhao Y, Zhao L, Wang H, Li Z, You Q and Guo Q: Wogonin induced G1 cell cycle arrest by regulating $\mathrm{Wnt} / \beta$-catenin signaling pathway and inactivating CDK8 in human colorectal cancer carcinoma cells. Toxicology 312: 36-47, 2013.

20. Gao J, Morgan WA, Sanchez-Medina A and Corcoran O: The ethanol extract of Scutellaria baicalensis and the active compounds induce cell cycle arrest and apoptosis including upregulation of p53 and Bax in human lung cancer cells. Toxicol Appl Pharmacol 254: 221-228, 2011

21. Dong P, Zhang Y, Gu J, Wu W, Li M, Yang J, Zhang L, Lu J, $\mathrm{Mu} \mathrm{J}$, Chen L, et al: Wogonin, an active ingredient of Chinese herb medicine Scutellaria baicalensis, inhibits the mobility and invasion of human gallbladder carcinoma GBC-SD cells by inducing the expression of maspin. J Ethnopharmacol 137: 1373-1380, 2011.

22. Huang KF, Zhang GD, Huang YQ and Diao Y: Wogonin induces apoptosis and down-regulates survivin in human breast cancer MCF-7 cells by modulating PI3K-AKT pathway. Int Immunopharmacol 12: 334-341, 2012.

23. Xu M, Lu N, Zhang H, Dai Q, Wei L, Li Z, You Q and Guo Q: Wogonin induced cytotoxicity in human hepatocellular carcinoma cells by activation of unfolded protein response and inactivation of AKT. Hepatol Res 43: 890-905, 2013.

24. Lin CC, Kuo CL, Lee MH, Lai KC, Lin JP, Yang JS, Yu CS, Lu CC, Chiang JH, Chueh FS, et al: Wogonin triggers apoptosis in human osteosarcoma U-2 OS cells through the endoplasmic reticulum stress, mitochondrial dysfunction and caspase-3-dependent signaling pathways. Int J Oncol 39: 217-224, 2011.

25. Lee DH, Lee TH, Jung $\mathrm{CH}$ and Kim YH: Wogonin induces apoptosis by activating the AMPK and p53 signaling pathways in human glioblastoma cells. Cell Signal 24: 2216-2225, 2012.

26. Tsai CF, Yeh WL, Huang SM, Tan TW and Lu DY: Wogonin induces reactive oxygen species production and cell apoptosis in human glioma cancer cells. Int J Mol Sci 13: 9877-9892, 2012.

27. Zhang M, Liu LP, Chen Y, Tian XY, Qin J, Wang D, Li Z and Mo SL: Wogonin induces apoptosis in RPMI 8226, a human myeloma cell line, by downregulating phospho-Akt and overexpressing Bax. Life Sci 92: 55-62, 2013.

28. Yang H, Hui H, Wang Q, Li H, Zhao K, Zhou Y, Zhu Y, Wang X, You Q, Guo Q, et al: Wogonin induces cell cycle arrest and erythroid differentiation in imatinib-resistant K562 cells and primary CML cells. Oncotarget 5: 8188-8201, 2014.
29. Xu X, Zhang Y, Li W, Miao H, Zhang H, Zhou Y, Li Z, You Q, Zhao L and Guo Q: Wogonin reverses multi-drug resistance of human myelogenous leukemia K562/A02 cells via downregulation of MRP1 expression by inhibiting Nrf2/ARE signaling pathway. Biochem Pharmacol 92: 220-234, 2014

30. Attia SM, Ahmad SF, Harisa GI, Mansour AM, El Sayed SM and Bakheet SA: Wogonin attenuates etoposide-induced oxidative DNA damage and apoptosis via suppression of oxidative DNA stress and modulation of OGG1 expression. Food Chem Toxicol 59: 724-730, 2013.

31. Masoud L, Vijayasarathy C, Fernandez-Cabezudo M, Petroianu G and Saleh AM: Effect of malathion on apoptosis of murine L929 fibroblasts: a possible mechanism for toxicity in low dose exposure. Toxicology 185: 89-102, 2003.

32. Alnemri ES: Mammalian cell death proteases: a family of highly conserved aspartate specific cysteine proteases. J Cell Biochem 64: 33-42, 1997.

33. Kuwana T and Newmeyer DD: Bcl-2-family proteins and the role of mitochondria in apoptosis. Curr Opin Cell Biol 15: 691-699, 2003.

34. Yang Y, Li XJ, Chen Z, Zhu XX, Wang J, Zhang LB, Qiang L, Ma YJ, Li ZY, Guo QL, et al: Wogonin induced calreticulin/ annexin A1 exposure dictates the immunogenicity of cancer cells in a PERK/AKT dependent manner. PLoS One 7: e50811, 2012.

35. Lindholm D, Wootz H and Korhonen L: ER stress and neurodegenerative diseases. Cell Death Differ 13: 385-392, 2006.

36. Zhou J, Liu CY, Back SH, Clark RL, Peisach D, Xu Z and Kaufman RJ: The crystal structure of human IRE1 luminal domain reveals a conserved dimerization interface required for activation of the unfolded protein response. Proc Natl Acad Sci USA 103: 14343-14348, 2006.

37. Winnay JN and Kahn CR: PI 3-kinase regulatory subunits as regulators of the unfolded protein response. Methods Enzymol 490: 147-158, 2011.

38. Rutkowski DT and Kaufman RJ: A trip to the ER: coping with stress. Trends Cell Biol 14: 20-28, 2004.

39. Schröder M and Kaufman RJ: ER stress and the unfolded protein response. Mutat Res 569: 29-63, 2005.

40. Pfaffenbach KT and Lee AS: The critical role of GRP78 in physiologic and pathologic stress. Curr Opin Cell Biol 23: 150-156, 2011.

41. Li J, Ni M, Lee B, Barron E, Hinton DR and Lee AS: The unfolded protein response regulator GRP78/BiP is required for endoplasmic reticulum integrity and stress-induced autophagy in mammalian cells. Cell Death Differ 15: 1460-1471, 2008.

42. Youle RJ and Strasser A: The BCL-2 protein family: opposing activities that mediate cell death. Nat Rev Mol Cell Biol 9: 47-59, 2008.

43. Lessene G, Czabotar PE and Colman PM: BCL-2 family antagonists for cancer therapy. Nat Rev Drug Discov 7: 989-1000, 2008.

44. Vogler M, Dinsdale D, Dyer MJ and Cohen GM: Bcl-2 inhibitors: small molecules with a big impact on cancer therapy. Cell Death Differ 16: 360-367, 2009.

45. Chung H, Jung YM, Shin DH, Lee JY, Oh MY, Kim HJ, Jang KS, Jeon SJ, Son KH and Kong G: Anticancer effects of wogonin in both estrogen receptor-positive and -negative human breast cancer cell lines in vitro and in nude mice xenografts. Int $\mathbf{J}$ Cancer 122: 816-822, 2008.

46. McCubrey JA, Lee JT, Steelman LS, Blalock WL, Moye PW, Chang F, Pearce M, Shelton JG, White MK, Franklin RA, et al: Interactions between the PI3K and Raf signaling pathways can result in the transformation of hematopoietic cells. Cancer Detect Prev 25: 375-393, 2001.

47. Hu P, Han Z, Couvillon AD and Exton JH: Critical role of endogenous Akt/IAPs and MEK1/ERK pathways in counteracting endoplasmic reticulum stress-induced cell death. J Biol Chem 279: 49420-49429, 2004.

48. Kim SJ, Kim HJ, Kim HR, Lee SH, Cho SD, Choi CS, Nam JS and Jung JY: Antitumor actions of baicalein and wogonin in HT-29 human colorectal cancer cells. Mol Med Rep 6: 1443-1449, 2012. 